\title{
Enhancement of properties in Mizar
}

\author{
Artur Korniłowicz ${ }^{\text {Corresp. } 1}$ \\ ${ }^{1}$ Institute of Computer Science, University of Bialystok, Bialystok, Poland \\ Corresponding Author: Artur Korniłowicz \\ Email address: arturk@math.uwb.edu.pl
}

A "property" in the Mizar proof-assistant is a construction that can be used to register chosen features of predicates (e.g. "reflexivity", "symmetry"), operations (e.g. "involutiveness", "commutativity") and types (e.g. "sethoodness") declared at the definition stage. The current implementation of Mizar allows using properties for notions with a specific number of visible arguments (e.g. reflexivity for a predicate with two visible arguments and involutiveness for an operation with just one visible argument).

In this paper we investigate a more general approach to overcome these limitations. We propose an extension of the Mizar language and a corresponding enhancement of the Mizar proof-checker which allow declaring properties of notions of arbitrary arity with respect to explicitly indicated arguments. Moreover, we introduce a new property -- the "fixedpoint-free" property of unary operations -- meaning that the result of applying the operation to its argument always differs from the argument.

Results of tests conducted on the Mizar Mathematical Library are presented. 


\title{
Enhancement of properties in Mizar
}

\section{Artur Korniłowicz ${ }^{1}$}

\author{
${ }^{1}$ Institute of Computer Science, University of Bialystok, Poland, \\ arturk@math.uwb.edu.pl, http://math.uwb.edu.pl/ arturk \\ Corresponding author: \\ Artur Korniłowicz ${ }^{1}$ \\ Email address: arturk@math.uwb.edu.pl
}

\section{\& ABSTRACT}

A property in the Mizar proof-assistant is a construction that can be used to register chosen features of predicates (e.g. "reflexivity", "symmetry"), operations (e.g. "involutiveness", "commutativity") and types (e.g. "sethoodness") declared at the definition stage. The current implementation of Mizar allows using properties for notions with a specific number of visible arguments (e.g. reflexivity for a predicate with two visible arguments and involutiveness for an operation with just one visible argument).

In this paper we investigate a more general approach to overcome these limitations. We propose an extension of the Mizar language and a corresponding enhancement of the Mizar proof-checker which allow declaring properties of notions of arbitrary arity with respect to explicitly indicated arguments. Moreover, we introduce a new property - the "fixedpoint-free" property of unary operations - meaning that the result of applying the operation to its argument always differs from the argument.

Results of tests conducted on the Mizar Mathematical Library are presented.

\section{INTRODUCTION}

Classical mathematical papers consist of sequences of definitions and justified facts classified into several categories like: theorems, lemmas, corollaries, and so on, often interspersed with some examples and descriptions.

Mathematical documents prepared using various proof-assistants (e.g., Isabelle (Isabelle, 2020), HOL Light (HOL Light, 2020), Coq (Coq, 2020), Metamath (Metamath, 2020), Lean (Lean, 2020), and Mizar (Mizar, 2020)) can also contain other constructions that are processable by dedicated software. In the case of the Mizar system (Bancerek et al., 2015; Grabowski et al., 2010) such constructions are:

1. existential, conditional and functorial registrations which enhance processing adjectives (Naumowicz, 2009),

2. term reductions which reduce terms to their proper sub-terms (Korniłowicz, 2013),

3. term identifications which identify equivalent notions from different theories (Grabowski et al., 2010), and

4. properties which can declare chosen properties of predicates, functors and types at the stage of their definitions (Naumowicz \& Byliński, 2004).

The current implementation of the Mizar proof-assistant allows using properties for notions with a specific number of visible arguments. Visible arguments are those which are explicitly used in the notation of the notion. For example, if $x$ and $y$ are elements of a group $G$, then for the operation $x+y$, where + denotes the addition of elements of the group $G, x$ and $y$ are visible arguments, while $G$ is a hidden argument of the operation.

In this paper we propose an extension of both the Mizar language and the Mizar proof-checker which allows declaring properties of notions of arbitrary arity with respect to explicitly indicated arguments. We also introduce a new property - the "fixedpoint-free" property of unary operations. It states that the result of applying the operation to its argument always differs from the argument.

The structure of the paper is the following: in Section 2 we present the Mizar proof-assistant with the focus on its features related to the new development proposed in this paper; in Section 3 we describe how to define and use properties for arbitrary arguments; in Section 4 we present the "fixedpoint-free" property; 
and finally, in Section 5, we describe some conclusions and plans for next enhancements of properties in Mizar. The results of implementing new features in the Mizar Mathematical Library (MML) (Bancerek et al., 2018; Alama et al., 2011) are shown in both Sections 3 and 4.

\section{MIZAR PROOF-ASSISTANT}

The Mizar project started in 1973 under the leadership of Andrzej Trybulec (Matuszewski \& Rudnicki, 2005; Grabowski et al., 2015). The main goal of the project is to develop a computer framework that allows writing mathematical papers under the control of computer programs that check syntactical, semantical and logical correctness of texts. The Mizar project consists of three main components:

- a language invented to write mathematical texts to be processed by computers,

- a collection of computer programs designed and implemented for processing texts written in the Mizar language, with its core program, a proof-checker named VERIFIER, suitable for formal verification (Avigad \& Harrison, 2014; Trybulec et al., 2013; Wiedijk, 2006), and

- the Mizar Mathematical Library - a library of documents (called articles) written in the Mizar language and verified by the Mizar proof-checker.

\subsection{Language}

The Mizar language reflects the natural language of mathematics and enables computers to efficiently process documents written in the language. It implements rules for writing: formulae of various kinds, definitions, theorems, local lemmas, reasoning methods, proof steps, and other syntactic constructions instructing the proof-checker to launch dedicated algorithms for processing particular mechanisms (e.g. term identifications (Grabowski et al., 2010), term reductions (Korniłowicz, 2013), properties of predicates and functors (Naumowicz \& Byliński, 2004)), etc.

For the purposes of this paper we recall some basic information about how new mathematical notions can be defined in Mizar articles.

The Mizar language allows users to define predicates, functors (linguistic functions used to define operations), attributes (Naumowicz, 2009), types (Bancerek, 2003), and structures. The general form of a definition consists of the definition arguments, permissive assumptions (necessary to prove the correctness of the definition), its notation (prefix, infix or suffix), the result type, the definiens, correctness conditions, and some extra properties. For example, the union of two sets can be defined as follows:

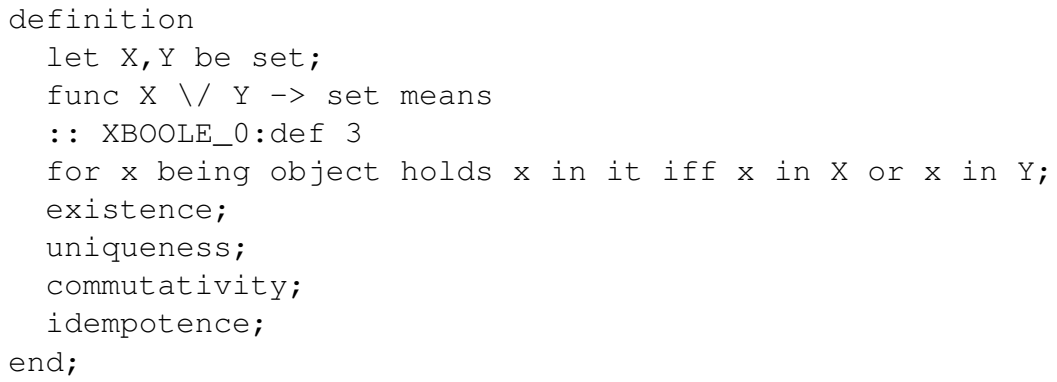

where the statement let $X, Y$ be set; introduces two arguments, func declares that it is a definition of an operation, $X \backslash / Y$ introduces the symbol $\backslash /$ for the union and declares it to be used as an infix symbol, $->$ set defines the type of the result of the operation (the union of two sets is a set), XBOOLE_O : def 3 is a unique identifier of the definition (it can be used to refer to the definition), for statement describes the meaning of the definition (it keyword in the definiens represents the notion being defined), existence is an automatically generated statement that has to be proved by authors to justify that there exists an object satisfying the definiens, uniqueness is another automatically generated statement that has to be proved to justify that there exists only one object satisfying the definiens, commutativity and idempotence are extra properties that can be declared and proved about the notion at this stage.

One can observe that in the above example definition there are no permissive assumptions, because they are not necessary to justify the existence and uniqueness. But, for example, in the definition of a homeomorphism between two topological structures: 


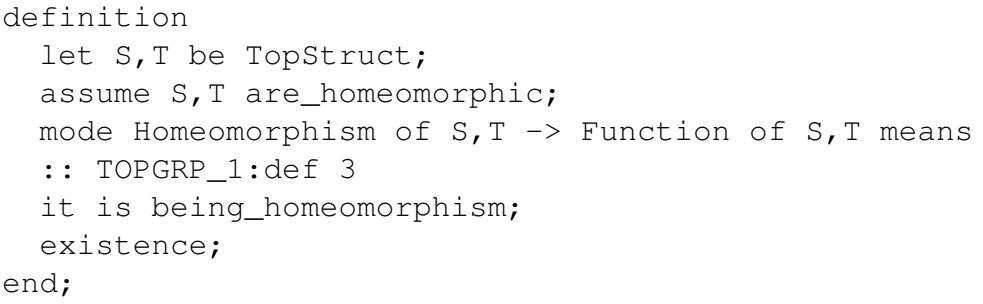

such an assumption assume S, T are_homeomorphic; is necessary to justify the existence, because in general not all topological spaces are homeomorphic.

The Mizar language allows also introducing, so called, redefinitions. Redefinitions can be used for the following purposes:

- to change result types of operations for more specific types of arguments; for example, addition of numbers can be defined for complex numbers with the result type representing complex numbers, but when arguments of the definition are, say, natural numbers, the result type of the addition can be redefined to the type representing natural numbers;

- to reformulate definiens formulae in domain languages adequate to the types of arguments of the notion; for example, the inclusion of arbitrary sets can be defined in terms of elements of the sets, while when arguments of the inclusion are binary relations, the definiens of the inclusion can be formulated in terms of pairs of elements.

\subsection{Proof checker}

The logical foundation of the Mizar checker is classical first-order logic with equality (in some contexts, however, free second-order variables are permitted enabling the introduction of schemes, e.g. the scheme of mathematical induction). The proof system is based on the Jaśkowski style of natural deduction (Jaśkowski, 1934). Structures of proofs are basically related to the structures of the formulae being proved with application of definitional expansions.

From the author's perspective, the correctness of formalized reasoning is controlled by the core utility of the Mizar system, the VERIFIER. Although its proof-checking code is sufficient to guarantee logical correctness, there are successful applications of external software to perform some particular tasks during processing Mizar texts (Naumowicz, 2015, 2014, 2010).

VERIFIER is a classical proof checker based on the notion of the inference obviousness (Davis, 1981; Rudnicki, 1987). The basic modules of VERIFIER are the following:

PARSER which is responsible for controlling the lexical structure of a given text and generating the parse tree of the text.

MSM PrOCESSOR which identifies constants, variables and labels.

ANALYZER which identifies objects and operations, performs type checking and resolves possible ambiguities caused by overloading of symbols. Moreover, it verifies if constraints required by particular constructions are fulfilled.

REASONER which controls structures of proofs according to the natural deduction rules.

CHECKER which verifies logical correctness of inferences. As a disprover it tries to refute negations of processed sentences. It performs propositional calculus (PRECHECKER), equational calculus over equalities accessible in inferences (EQUALIZER) and unification (UNIFIER).

\subsection{Mizar Mathematical Library}

The Mizar Mathematical Library (MML) (Bancerek et al., 2018; Alama et al., 2011) was established in 1989 to accumulate mathematical knowledge formalized and verified using the Mizar proof-assistant. It is a collection of papers based on the Tarski-Grothendieck (TG) set theory, which is a variant of the ZFC set theory (Hayden et al., 1968), where the axiom of infinity is replaced by Tarski's axiom of existence of arbitrarily large, strongly inaccessible cardinals (Tarski, 1939).

The current Version 5.63.1382 of the Mizar Mathematical Library contains 1385 articles (107,756,687 bytes in total) devoted to various branches of mathematics.

Developing the MML includes the following tasks: 
- collecting new knowledge realized by: a) developing background knowledge to prepare a comprehensive database for practicing mathematicians and for educational purposes; b) formalizing entire mathematical books (Gierz et al., 1980; Bancerek \& Rudnicki, 2002); c) formalizing wellknown theorems (Abad \& Abad, 1999); and d) developing new theories (Grabowski, 2014, 2013; Grabowski \& Jastrzębska, 2010).

- refactoring the database (Grabowski \& Schwarzweller, 2007) to keep its integrity (Rudnicki \& Trybulec, 2003) and to increase readability of the stored proofs (Pąk, 2014).

Knowledge stored in the database is used in various branches of science and education, e.g. for representing mathematics on WWW (Iancu et al., 2013; Urban, 2005), as an input for ATP systems (Urban et al., 2013; Urban \& Sutcliffe, 2010; Urban et al., 2010; Urban, 2008), as an input for services classifying mathematics (Grabowski \& Schwarzweller, 2012), and others.

\subsection{Processing Mizar articles}

Every Mizar article written as a plain text file with the file extension . mi z consists of two main parts: its environment, which can be seen as the import part from the Mizar Mathematical Library, and text-proper part, where new definitions, lemmas, theorems etc. are placed.

In the environment part the following directives are allowed:

- vocabularies - imports symbols of notions stored in the MML.

- notations - imports notations of notions stored in the MML. The order is important - in the case of overloading the last one counts.

- constructors - imports constructors (meanings) of notions.

- theorems - imports theorems to which proofs refer to.

- schemes - imports schemes to which proofs refer to.

- definitions - imports formulae that determine proof skeletons.

- registrations - imports registrations, term identifications and term reductions used in proofs.

- equalities - imports equalities of operations defined using equals clause with their meanings.

- expansions - imports expansions of predicates and adjectives.

- requirements - imports switches to launch build-in procedures by the checker.

The environment is processed by a dedicated program - ACCOMMODATOR. It reads the environment part of the article and prepares global notions ready to be used in the local article. When it is done, VERIFIER processes the text-proper part of the article. Firstly, PARSER scans the article, checks its grammatical correctness and prepares the parse tree of the article. The parse tree is stored in the XML file with the extension . ws (Naumowicz \& Piliszek, 2016). The next submodule, MSM ProcESSOR, reads the .wsx file and identifies all identifiers of constants, variables and labels that appear in the article. MSM PROCESSOR adds computed information to data written in the . wSX file and creates another XML file with the extension .msx. Then, ANALYZER reads the .msx file and resolves ambiguities and identifies used notions (predicates, adjectives, types, operations and structures). ANALYZER creates another XML file with the extension . xml - the structure of this . xml file differs from structures of both . ws $x$ and .msx files. The . xml file contains the complete semantic information about all constructions used in the processed article. When all ambiguities are resolved and all notions are identified, the article is ready to be verified against its logical correctness by the Mizar checker. Formulae are negated, transformed to their disjunctive normal forms and all disjuncts, one by one, are then verified by EQUALIZER - a Mizar module dealing with equational calculus (Rudnicki \& Trybulec, 2001). It collects all terms from the processed disjunct, and computes the congruence closure over equalities available in the inference. The equalities can be provided by various Mizar constructions, like: term expansions (equals), properties of operations, term reductions, term identifications, arithmetic, type changing (reconsider), and others, e.g. processing structures.

For the sake of this paper let us underline properties of operations. They are described in more detail in Section 3.

The last procedure applied to the processed inference is its unification. If EQUALIZER cannot disprove the formula, UNIFIER starts working and tries to refute it. If UNIFIER finds a contradiction, the original disjunct is accepted as true; otherwise, appropriate messages are reported and authors are supposed to complete missing proofs. 
When all formulae are accepted, the article can be submitted to the Mizar Mathematical Library and the new knowledge can be used in subsequent works. Two other tools are used to export the new article into the database: EXPORTER - extracts public knowledge from the article, and TRANSFERER - transfers the knowledge into the Mizar Mathematical Library.

\section{PROPERTIES}

Properties in Mizar are constructions which can be used to declare that predicates are reflexive $\left(\forall_{x}: x R x\right)$, irreflexive $\left(\forall_{x}: \neg x R x\right)$, symmetric $\left(\forall_{x, y}: x R y \rightarrow y R x\right)$, asymmetric $\left(\forall_{x, y}: x R y \rightarrow \neg y R x\right)$, and connected $\left(\forall_{x, y}: x R y \vee y R x\right)$; in the case of operations, they can be declared as involutive $(f(f(x))=x)$, projective $(f(f(x))=f(x))$, idempotent $(f(x, x)=x)$, and commutative $(f(x, y)=f(y, x))$. Such declarations of chosen properties must be placed within definitional blocks. When a notion is equipped with some properties, then adequate formulae involving the notion become obvious to the Mizar checker without any explicit reference to the definition and any theorem (they are processed automatically based on internally generated equalities of terms in cases of properties of functors and appropriate formulae in cases of properties of predicates). For example, the declaration of the idempotence of the binary union of sets implies that the equality $A \cup A=A$ becomes obvious for any set $A$.

The current implementation of the Mizar checker is restricted to fixed numbers of visible arguments of considered notions listed in Table 1.

\begin{tabular}{|c|c|c|c|}
\hline \multicolumn{2}{|c|}{ Predicates } & \multicolumn{2}{c|}{ Functors } \\
\hline property name & arity & property name & arity \\
\hline reflexivity & 2 & projectivity & 1 \\
\hline irreflexivity & 2 & involutiveness & 1 \\
\hline symmetry & 2 & commutativity & 2 \\
\hline asymmetry & 2 & idempotence & 2 \\
\hline connectedness & 2 & & \\
\hline
\end{tabular}

Table 1. Arities of properties of notions

In this work we propose an extension of the Mizar system with the possibility of explicit indication with respect to which visible arguments of mathematical notions given properties can be declared. To achieve this, one can use the wrt clause followed by a comma separated list_of_loci of visible arguments of lengths presented in Table 1. The extended syntax of a definition of a functor is the following:

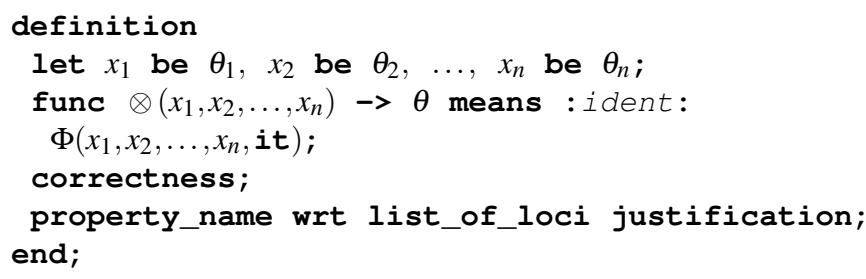

and the extended syntax of a definition of a predicate is the following:

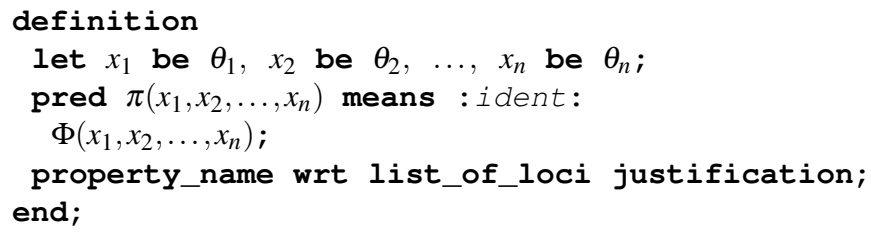

For the back compatibility the wrt clause is not obligatory, definitions with no wrt clause work as in previous releases of the Mizar checker. 


\subsection{Examples}

As an example of using this new feature in the MML we can cite the theorem (Kusak \& Radziszewski, 1991):

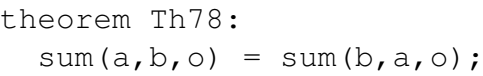

which can be reformulated as commutativity of:

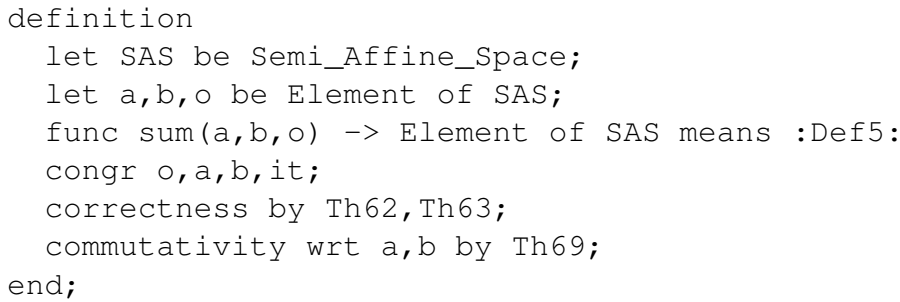

An interesting example of a theorem which at the first glance looks like symmetry of some quaternary predicate with respect to the third and forth argument, but cannot be reformulated as symmetry of the predicate, is (Oryszczyszyn \& Prażmowski, 1990):

theorem Th24:

p, q _ _ p1,q1 implies p, q _ _ q1,p1;

To explain this fact one should look at types of variables $p, q, p 1$, and $q 1$ used in the theorem and types of arguments of the definition of the predicate $\_$l_. The type of $p, q, p 1$, and $q 1$ is

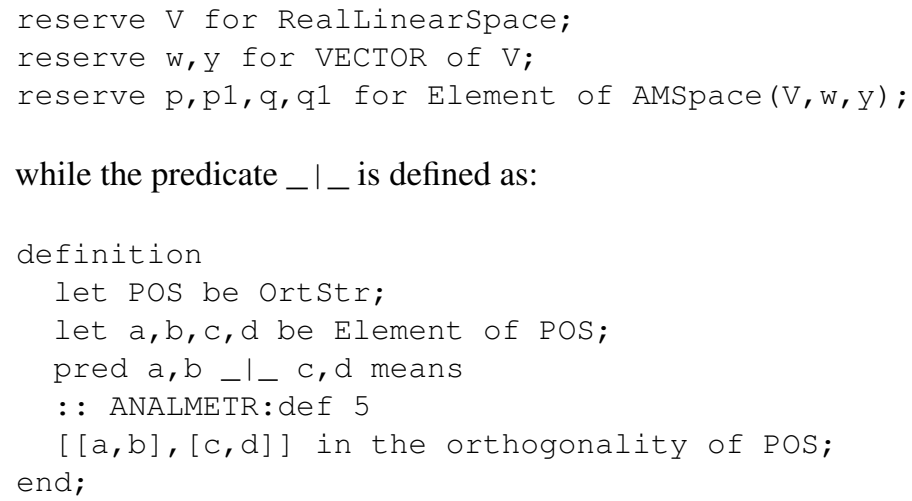

Now it is clear that types of variables $p, q, p 1$, and $q 1$ are more restricted than original types of arguments $a, b, c$, and $d$ of the predicate declared in its definition. The statement proved as the mentioned theorem Th2 4 is true for elements of a particular space AMSpace $(V, w, y)$, but does not hold for elements of an arbitrary space OrtStr.

It is a very typical case, when some notion is defined for general types of arguments, and its particular properties are provable for less general ones.

\subsection{Changes in XML files}

As it was said in Section 2.4, the Mizar verifier, to check the correctness of Mizar articles, generates and processes several intermediate files written in XML formats. To be able to implement the feature considered in this section, we had to slightly change the grammars of these XML files. From the perspective of Mizar users formalizing some knowledge, these changes are not important - the authors are not supposed to look into these files. For researchers who use the Mizar system for other purposes and develop external applications working on the semantic level of the Mizar Mathematical Library (Urban, 2005), these changes will induce the need for some adjustments or reimplementations. Therefore, below we explain the changes. Let us take the following definition: 


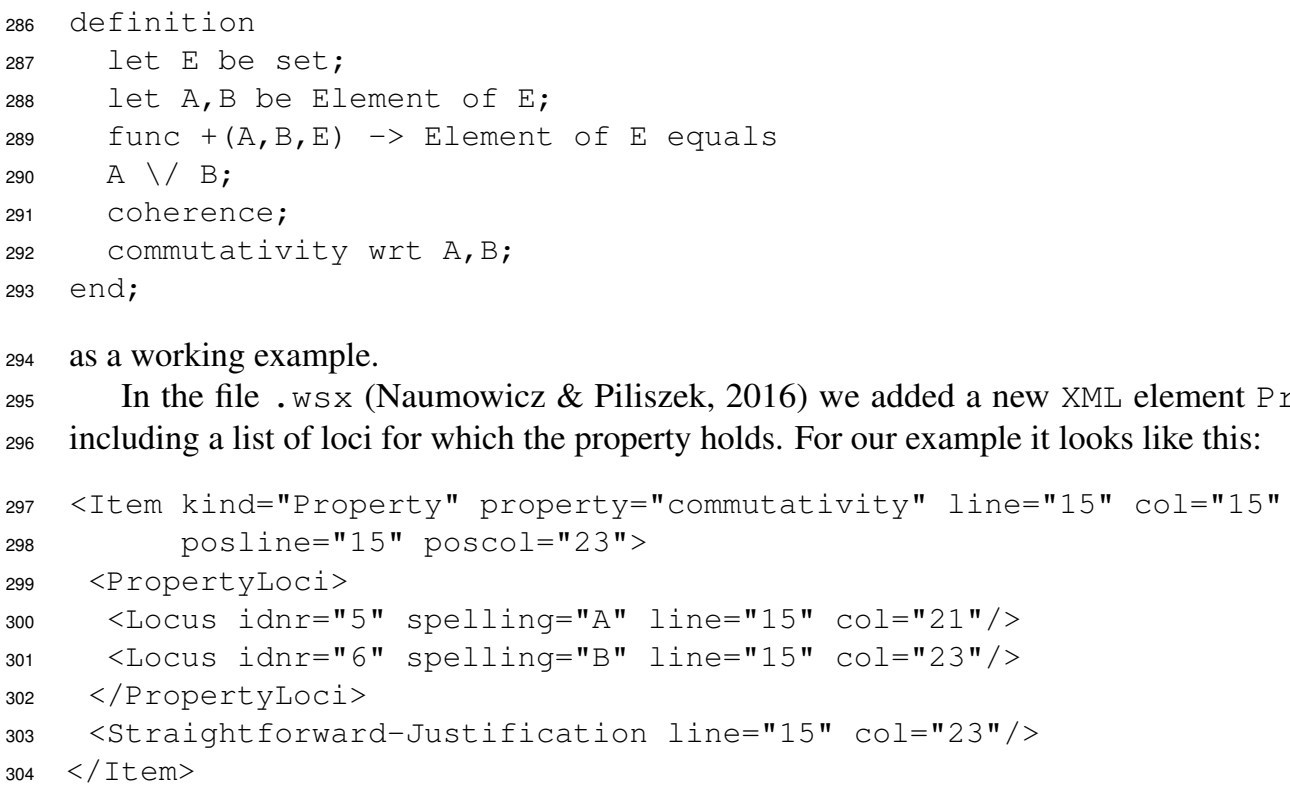

This is propagated to the .msx file, which is an extension of the .wsx file, and for our example it becomes:

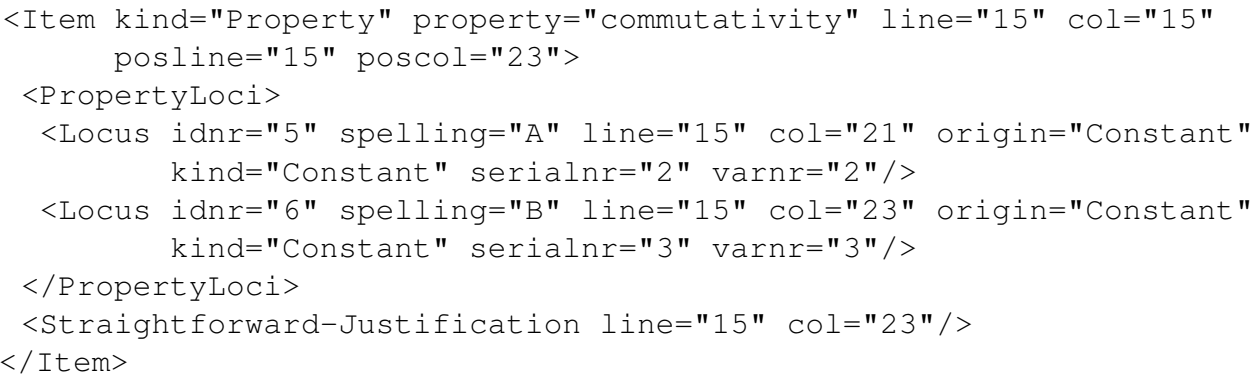

Next two changes are introduced in .xml files: we added internal descriptions of properties in their definitions:

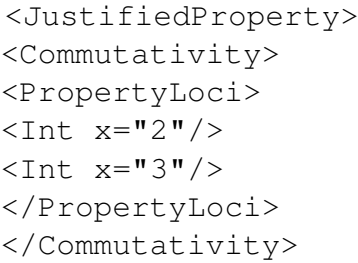

and in constructors with which properties are associated:

<Constructor kind="K" nr="1" aid="EE" relnr="20">

$<$ Properties $>$

$<$ Commutativity propertyarg1="2" propertyarg2="3"/>

$</$ Properties $>$

\section{FIXEDPOINT-FREE PROPERTY}

As another enhancement of properties in Mizar we propose a new unary property of functors - "fixedpointfree". The fixedpoint-free property is meaningful for operations of which the result of application to a given argument is always different from the argument. This is reflected in justification formulae of the properties to be proved at the stage of defining the operation.

We propose the following syntax and formulae to be proved to justify the correctness of the property for given functors. In the case of functors defined using the means clause with a simple definiens it is: 


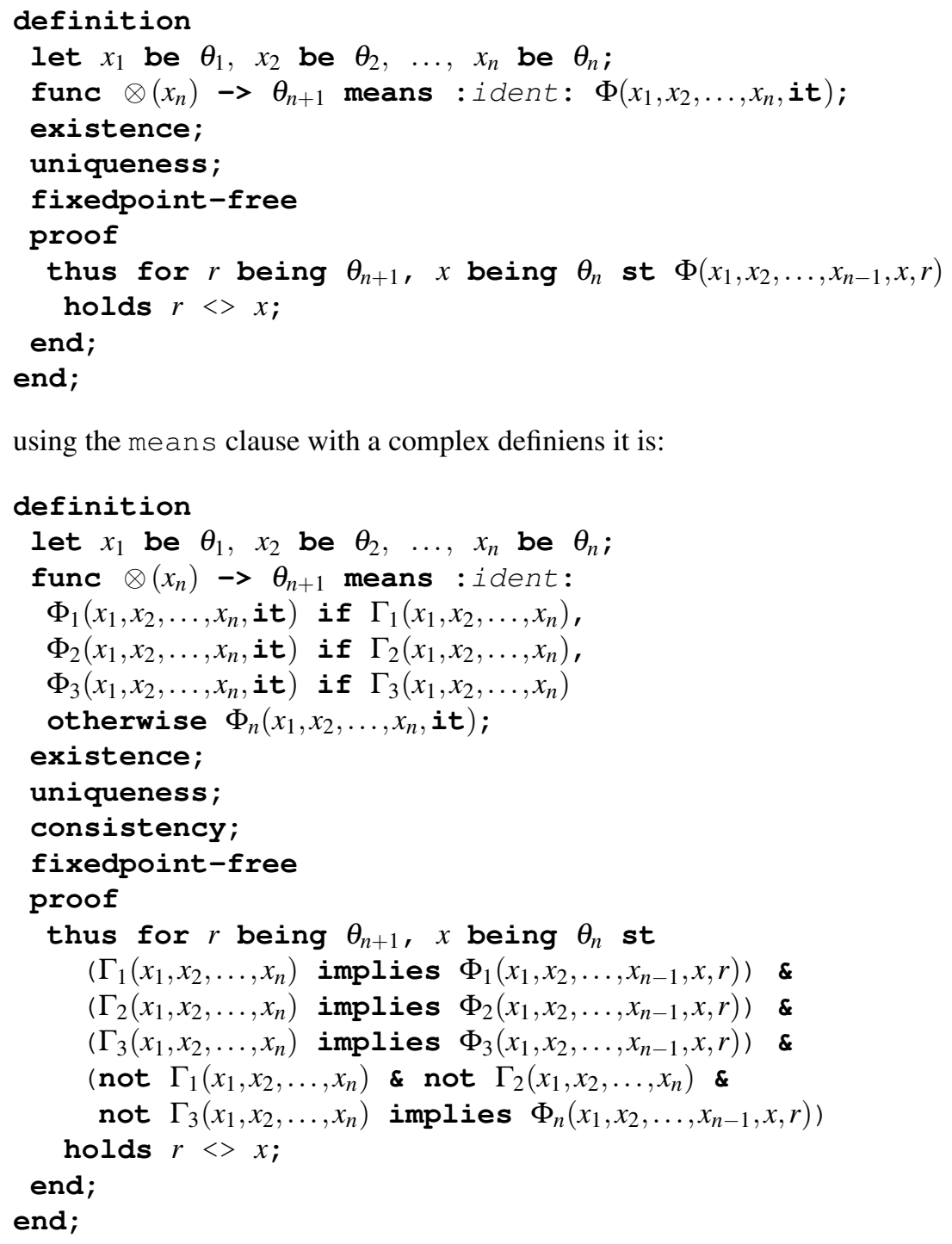




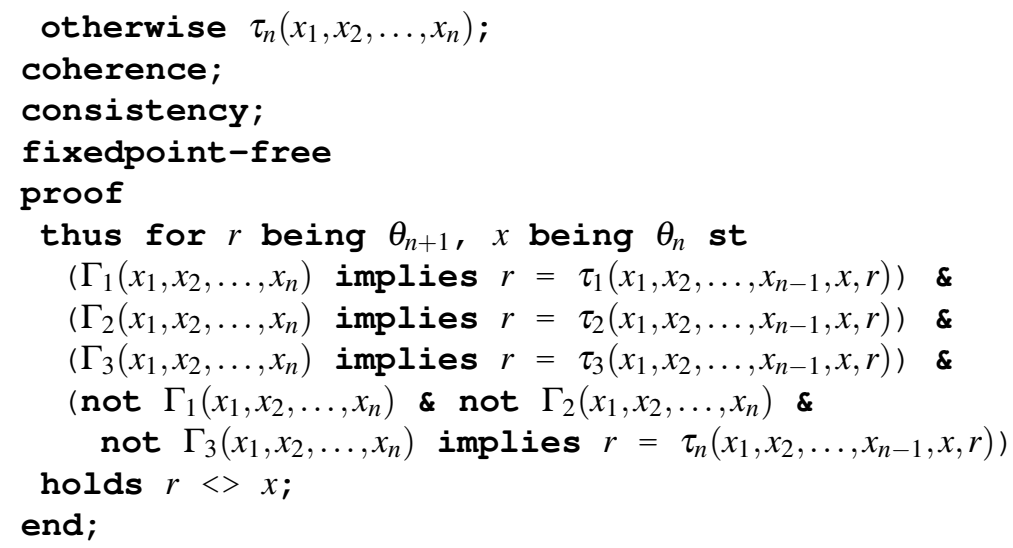

This property can also be declared with the wrt clause as described in Section 3, and could be added to Table 1.

An important part of our work was implementing a tool (FIXEDPOINTFREEDETECTOR) which detects MML theorems that could be rewritten as fixedpoint-free properties of operations used in formulations of the theorems. To detect such theorems, the following steps should be done:

1. If the theorem is a conjunction of some atomic formulae, decomposing a given formula to a list of atomic formulae.

2. Selecting inequalities among the atomic formulae.

3. Selecting formulae which compare unary terms with single variables among the inequalities.

4. For each such a formula checking whether:

(a) the argument of the unary term equals to a single variable,

(b) the type of the variable and the type of the argument of the term declared in the definition of the operation are equal.

5. If both answers to the above questions (4a) and (4b) are positive, marking the fact to be replaceable by the fixedpoint-free property of the operation.

At the end of this section we present results of launching the detector on the Mizar Mathematical Library. In the current version of the library 3 such theorems were found in 3 articles. They are: the power set of a set, the successor of a set, and poles at infinity of elements of the absolute. Changing the theorems to the properties caused that 10 inferences in the Mizar Mathematical Library became obvious. Even though these numbers obtained in tests are not too big, the Library Committee of the Mizar project will analyze the cases and will decide about incorporating them into the library. In the case of approval, a refactoring (Grabowski \& Schwarzweller, 2007) of the MML will be processed.

Computations were carried out at the Computer Center of University of Białystok http: / / uco. uwb.edu.pl

\section{CONCLUSIONS AND FUTURE WORK}

Although the basic concept of properties had been introduced to quite early releases of the Mizar system, we still see possibilities to design and develop new features of properties in Mizar. In this paper we described two new features: a) we presented the syntax and semantics of a new property (fixedpoint-free) which enriches the Mizar language and increases the computational power of the Mizar checker by a more automatic processing of unary operations with no fixed points, and b) we removed a restriction on the application of already defined properties for fixed positions of visible arguments. Investigating a more general approach to introducing properties resulted in an extension of the Mizar language and a corresponding enhancement of the Mizar proof-checker. To analyze the potential usefulness of the proposed general approach we implemented a dedicated software tool and conducted appropriate tests with it on the current Mizar Mathematical Library.

As future work, we plan to open the system of properties for arbitrary (when possible) arities of predicates and functors. We already see within the current content of the Mizar Mathematical Library 
several applications of that approach, e.g. we would be able to define commutativity for enumerated sets with cardinality greater than two, reflexivity and symmetry of the relation of lying more than two points on a given line, and others. We also plan to investigate new sorts of properties, like associativity or being one-to-one of functors.

\section{REFERENCES}

Abad, P. \& Abad, J. (1999). The Hundred Greatest Theorems http://www.cs.ru.nl/ freek/ $100 \%$

Alama, J., Kohlhase, M., Mamane, L., Naumowicz, A., Rudnicki, P., \& Urban, J. (2011). Licensing the Mizar Mathematical Library. In J. H. Davenport, W. M. Farmer, J. Urban, \& F. Rabe (Eds.), Proceedings of the 18th Calculemus and 10th International Conference on Intelligent Computer Mathematics, volume 6824 of MKM'11, Lecture Notes in Computer Science (pp. 149-163). Berlin, Heidelberg: Springer-Verlag. http://dx.doi.org/10.1007/978-3-642-22673-1_11.

Avigad, J. \& Harrison, J. (2014). Formally Verified Mathematics. Communications of the ACM, 57(4), 66-75. https://doi.org/10.1145/2591012.

Bancerek, G. (2003). On the Structure of Mizar Types. In H. Geuvers \& F. Kamareddine (Eds.), Electronic Notes in Theoretical Computer Science, volume 85 (pp. 69-85).: Elsevier.

Bancerek, G., Byliński, C., Grabowski, A., Korniłowicz, A., Matuszewski, R., Naumowicz, A., \& Pąk, K. (2018). The Role of the Mizar Mathematical Library for Interactive Proof Development in Mizar. Journal of Automated Reasoning, 61(1), 9-32. https://doi .org/10.1007/ s10817-017-9440-6.

Bancerek, G., Byliński, C., Grabowski, A., Korniłowicz, A., Matuszewski, R., Naumowicz, A., Pąk, K., \& Urban, J. (2015). Mizar: State-of-the-art and Beyond. In M. Kerber, J. Carette, C. Kaliszyk, F. Rabe, \& V. Sorge (Eds.), Intelligent Computer Mathematics - International Conference, CICM 2015, Washington, DC, USA, July 13-17, 2015, Proceedings, volume 9150 of Lecture Notes in Computer Science (pp. 261-279):: Springer. http://dx.doi.org/10.1007/978-3-319-20615-8_17.

Bancerek, G. \& Rudnicki, P. (2002). A Compendium of Continuous Lattices in Mizar: Formalizing Recent Mathematics. Journal of Automated Reasoning, 29(3-4), 189-224.

Coq (2020). Coq. https: //coq.inria.fr/.

Davis, M. (1981). Obvious logical inferences. In Proceedings of the Seventh International Joint Conference on Artificial Intelligence (pp. 530-531).

Gierz, G., Hofmann, K., Keimel, K., Lawson, J., Mislove, M., \& Scott, D. (1980). A Compendium of Continuous Lattices. Berlin, Heidelberg, New York: Springer-Verlag.

Grabowski, A. (2013). Automated Discovery of Properties of Rough Sets. Fundamenta Informaticae, 128(1-2), 65-79. https://doi.org/10.3233/FI-2013-933.

Grabowski, A. (2014). Efficient Rough Set Theory Merging. Fundamenta Informaticae, 135(4), 371-385. https://doi.org/10.3233/FI-2014-1129.

Grabowski, A. \& Jastrzębska, M. (2010). A Note on a Formal Approach to Rough Operators. In M. S. Szczuka, M. Kryszkiewicz, S. Ramanna, R. Jensen, \& Q. Hu (Eds.), Rough Sets and Current Trends in Computing - 7th International Conference, RSCTC 2010, Warsaw, Poland, June 28-30, 2010. Proceedings, volume 6086 of Lecture Notes in Computer Science (pp. 307-316).: Springer. http://dx.doi.org/10.1007/978-3-642-13529-3_33.

Grabowski, A., Korniłowicz, A., \& Naumowicz, A. (2010). Mizar in a Nutshell. Journal of Formalized Reasoning, Special Issue: User Tutorials I, 3(2), 153-245. https://doi.org/10.6092/issn. 1972-5787/1980.

Grabowski, A., Korniłowicz, A., \& Naumowicz, A. (2015). Four Decades of Mizar. Journal of Automated Reasoning, 55(3), 191-198. https://doi.org/10.1007/s10817-015-9345-1.

Grabowski, A. \& Schwarzweller, C. (2007). Revisions as an Essential Tool to Maintain Mathematical Repositories. In Proceedings of the 14th Symposium on Towards Mechanized Mathematical Assistants: 6th International Conference, Calculemus '07 / MKM '07 (pp. 235-249). Berlin, Heidelberg: SpringerVerlag. http://dx.doi.org/10.1007/978-3-540-73086-6_20.

Grabowski, A. \& Schwarzweller, C. (2012). Towards Automatically Categorizing Mathematical Knowledge. In M. Ganzha, L. A. Maciaszek, \& M. Paprzycki (Eds.), Federated Conference on Computer Science and Information Systems - FedCSIS 2012, Wroclaw, Poland, 9-12 September 2012, Proceedings (pp. 63-68). 
Hayden, S., Fraenkel, A. A., Zermelo, E., \& Kennison, J. F. (1968). Zermelo-Fraenkel Set Theory by Seymour Hayden and John F. Kennison. C. E. Merrill Columbus, Ohio.

HOL Light (2020). HOL Light. https: / /www. cl. cam.ac.uk/ jrh13/hol-light/. Iancu, M., Kohlhase, M., Rabe, F., \& Urban, J. (2013). The Mizar Mathematical Library in OMDoc: Translation and Applications. Journal of Automated Reasoning, 50(2), 191-202. https://doi. org/10.1007/s10817-012-9271-4.

Isabelle (2020). Isabelle. https: / / isabelle.in.tum.de/.

Jaśkowski, S. (1934). On the Rules of Suppositions in Formal Logic. Studia Logica. Nakładem Seminarjum Filozoficznego Wydziału Matematyczno-Przyrodniczego Uniwersytetu Warszawskiego http: / /books.google.pl/books?id=6w0vRAAACAAJ.

Korniłowicz, A. (2013). On Rewriting Rules in Mizar. Journal of Automated Reasoning, 50(2), 203-210. https://doi.org/10.1007/s10817-012-9261-6.

Kusak, E. \& Radziszewski, K. (1991). Semi_Affine Space. Formalized Mathematics, 2(3), 349-356. http://fm.mizar.org/1991-2/pdf2-3/semi_af1.pdf.

Lean (2020). Lean. https:// leanprover.github.io/.

Matuszewski, R. \& Rudnicki, P. (2005). Mizar: The First 30 Years. Mechanized Mathematics and Its Applications, Special Issue on 30 Years of Mizar, 4(1), 3-24.

Metamath (2020). Metamath. http://us.metamath.org/mpegif/mmset.html.

Mizar (2020). Mizar. http: //mizar.uwb edu.pl/.

Naumowicz, A. (2009). Enhanced Processing of Adjectives in Mizar. In A. Grabowski \& A. Naumowicz (Eds.), Computer Reconstruction of the Body of Mathematics, volume 18(31) of Studies in Logic, Grammar and Rhetoric (pp. 89-101). University of Białystok.

Naumowicz, A. (2010). Interfacing External CA Systems for Gröbner Bases Computation in Mizar Proof Checking. International Journal of Computer Mathematics, 87(1), 1-11. https://doi . org/10. $1080 / 00207160701864459$.

Naumowicz, A. (2014). SAT-Enhanced Mizar Proof Checking. In S. M. Watt, J. H. Davenport, A. P. Sexton, P. Sojka, \& J. Urban (Eds.), Intelligent Computer Mathematics - International Conference, CICM 2014, Coimbra, Portugal, July 7-11, 2014. Proceedings, volume 8543 of Lecture Notes in Computer Science (pp. 449-452).: Springer. http://dx.doi.org/10.1007/978-3-319-08434-3_37.

Naumowicz, A. (2015). Automating Boolean Set Operations in Mizar Proof Checking with the Aid of an External SAT Solver. Journal of Automated Reasoning, 55(3), 285-294. https: // doi . org/10. $1007 /$ s10817-015-9332-6.

Naumowicz, A. \& Byliński, C. (2004). Improving Mizar Texts with Properties and Requirements. In A. Asperti, G. Bancerek, \& A. Trybulec (Eds.), Mathematical Knowledge Management, Third International Conference, MKM 2004 Proceedings, volume 3119 of MKM'04, Lecture Notes in Computer Science (pp. 290-301). http://dx.doi.org/10.1007/978-3-540-27818-4_21.

Naumowicz, A. \& Piliszek, R. (2016). Accessing the Mizar Library with a Weakly Strict Mizar Parser. In M. Kohlhase, M. Johansson, B. R. Miller, L. de Moura, \& F. W. Tompa (Eds.), Intelligent Computer Mathematics - 9th International Conference, CICM 2016, Bialystok, Poland, July 25-29, 2016, Proceedings, volume 9791 of Lecture Notes in Computer Science (pp. 77-82).: Springer. http: //dx.doi.org/10.1007/978-3-319-42547-4_6.

Oryszczyszyn, H. \& Prażmowski, K. (1990). Analytical Metric Affine Spaces and Planes. Formalized Mathematics, 1(5), 891-899. http://fm.mizar.org/1990-1/pdf1-5/analmetr.pdf.

Pąk, K. (2014). Improving Legibility of Natural Deduction Proofs is Not Trivial. Logical Methods in Computer Science, 10(3), 1-30. https: / / doi .org/10.2168/LMCS-10 (3:23) 2014.

Rudnicki, P. (1987). Obvious Inferences. Journal of Automated Reasoning, 3(4), 383-393. https : //doi.org/http://dx.doi.org/10.1007/BF00247436.

Rudnicki, P. \& Trybulec, A. (2001). Mathematical Knowledge Management in Mizar. In Proc. of MKM 2001.

Rudnicki, P. \& Trybulec, A. (2003). On the Integrity of a Repository of Formal Mathematics. In A. Asperti, B. Buchberger, \& J. H. Davenport (Eds.), Proceedings of MKM-2003, volume 2594 of Lecture Notes in Computer Science (pp. 162-174).: Springer-Verlag Berlin Heidelberg.

Tarski, A. (1939). On Well-ordered Subsets of any Set. Fundamenta Mathematicae, 32, 176-183.

Trybulec, A., Korniłowicz, A., Naumowicz, A., \& Kuperberg, K. (2013). Formal Mathematics for Mathematicians. Journal of Automated Reasoning, 50(2), 119-121. https://doi.org/10. 
$549 \quad 1007 / \mathrm{s} 10817-012-9268-z$.

Urban, J. (2005). XML-izing Mizar: Making Semantic Processing and Presentation of MML Easy. In M. Kohlhase (Ed.), Mathematical Knowledge Management, 4th International Conference, MKM 2005, Bremen, Germany, July 15-17, 2005, Revised Selected Papers, volume 3863 of Lecture Notes in Computer Science (pp. 346-360):: Springer. http://dx.doi.org/10.1007/11618027_23.

Urban, J. (2008). Automated Reasoning for Mizar: Artificial Intelligence through Knowledge Exchange. In P. Rudnicki, G. Sutcliffe, B. Konev, R. A. Schmidt, \& S. Schulz (Eds.), Proceedings of the LPAR 2008 Workshops, Knowledge Exchange: Automated Provers and Proof Assistants, and the 7th International Workshop on the Implementation of Logics, Doha, Qatar, November 22, 2008, volume 418 of CEUR Workshop Proceedings: CEUR-WS.org http:// ceur-ws.org/Vol-418/paper1.pdf.

Urban, J., Hoder, K., \& Voronkov, A. (2010). Evaluation of Automated Theorem Proving on the Mizar Mathematical Library. In K. Fukuda, J. van der Hoeven, M. Joswig, \& N. Takayama (Eds.), Mathematical Software - ICMS 2010, Third International Congress on Mathematical Software, Kobe, Japan, September 13-17, 2010. Proceedings, volume 6327 of Lecture Notes in Computer Science (pp. 155-166):: Springer. http://dx.doi.org/10.1007/978-3-642-15582-6_30.

Urban, J., Rudnicki, P., \& Sutcliffe, G. (2013). ATP and Presentation Service for Mizar Formalizations. Journal of Automated Reasoning, 50(2), 229-241. https://doi.org/10.1007/ s10817-012-9269-y.

Urban, J. \& Sutcliffe, G. (2010). Automated Reasoning and Presentation Support for Formalizing Mathematics in Mizar. In S. Autexier, J. Calmet, D. Delahaye, P. D. F. Ion, L. Rideau, R. Rioboo, \& A. P. Sexton (Eds.), Intelligent Computer Mathematics, 10th International Conference, AISC 2010, 17th Symposium, Calculemus 2010, and 9th International Conference, MKM 2010, Paris, France, July 5-10, 2010. Proceedings, volume 6167 of Lecture Notes in Computer Science (pp. 132-146).: Springer. http://dx.doi.org/10.1007/978-3-642-14128-7_12.

Wiedijk, F., Ed. (2006). The Seventeen Provers of the World, Foreword by Dana S. Scott, volume 3600 of Lecture Notes in Computer Science. Springer. https : / doi .org/10.1007/11542384. 